ORIGINAL ARTICLE

\section{Tucatinib, Trastuzumab, and Capecitabine for HER2-Positive Metastatic Breast Cancer}

\author{
R.K. Murthy, S. Loi, A. Okines, E. Paplomata, E. Hamilton, S.A. Hurvitz, N.U. Lin, \\ V. Borges, V. Abramson, C. Anders, P.L. Bedard, M. Oliveira, E. Jakobsen, \\ T. Bachelot, S.S. Shachar, V. Müller, S. Braga, F.P. Duhoux, R. Greil, D. Cameron, \\ L.A. Carey, G. Curigliano, K. Gelmon, G. Hortobagyi, I. Krop, S. Loibl, M. Pegram, \\ D. Slamon, M.C. Palanca-Wessels, L. Walker, W. Feng, and E.P. Winer
}

A BSTRACT

\section{BACKGROUND}

Patients with human epidermal growth factor receptor 2 (HER2)-positive metastatic breast cancer who have disease progression after therapy with multiple HER2-targeted agents have limited treatment options. Tucatinib is an investigational, oral, highly selective inhibitor of the HER2 tyrosine kinase.

\section{METHODS}

We randomly assigned patients with HER2-positive metastatic breast cancer previously treated with trastuzumab, pertuzumab, and trastuzumab emtansine, who had or did not have brain metastases, to receive either tucatinib or placebo, in combination with trastuzumab and capecitabine. The primary end point was progression-free survival among the first 480 patients who underwent randomization. Secondary end points, assessed in the total population (612 patients), included overall survival, progression-free survival among patients with brain metastases, confirmed objective response rate, and safety.

\section{RESULTS}

Progression-free survival at 1 year was 33.1\% in the tucatinib-combination group and $12.3 \%$ in the placebo-combination group (hazard ratio for disease progression or death, $0.54 ; 95 \%$ confidence interval [CI], 0.42 to $0.71 ; \mathrm{P}<0.001$ ), and the median duration of progression-free survival was 7.8 months and 5.6 months, respectively. Overall survival at 2 years was $44.9 \%$ in the tucatinib-combination group and $26.6 \%$ in the placebocombination group (hazard ratio for death, $0.66 ; 95 \% \mathrm{CI}, 0.50$ to $0.88 ; \mathrm{P}=0.005$ ), and the median overall survival was 21.9 months and 17.4 months, respectively. Among the patients with brain metastases, progression-free survival at 1 year was $24.9 \%$ in the tucatinib-combination group and $0 \%$ in the placebo-combination group (hazard ratio, 0.48 ; $95 \% \mathrm{CI}, 0.34$ to 0.69 ; $\mathrm{P}<0.001$ ), and the median progression-free survival was 7.6 months and 5.4 months, respectively. Common adverse events in the tucatinib group included diarrhea, palmar-plantar erythrodysesthesia syndrome, nausea, fatigue, and vomiting. Diarrhea and elevated aminotransferase levels of grade 3 or higher were more common in the tucatinib-combination group than in the placebo-combination group.

\section{CONCLUSIONS}

In heavily pretreated patients with HER2-positive metastatic breast cancer, including those with brain metastases, adding tucatinib to trastuzumab and capecitabine resulted in better progression-free survival and overall survival outcomes than adding placebo; the risks of diarrhea and elevated aminotransferase levels were higher with tucatinib. (Funded by Seattle Genetics; HER2CLIMB ClinicalTrials.gov number, NCT02614794.)
The authors' full names, academic degrees, and affiliations are listed in the Appendix. Address reprint requests to Dr. Winer at the Dana-Farber Cancer Institute, 450 Brookline Ave., Boston, MA 02215, or at eric_winer@dfci.harvard.edu; or to Dr. Murthy at the University of Texas M.D. Anderson Cancer Center, 1515 Holcombe Blvd., Unit 1354, Houston, TX 77030, or at rmurthyl@mdanderson.org.

This article was published on December 11, 2019, at NEJM.org.

DOI: 10.1056/NEJMoa1914609 Copyright (@) 2019 Massachusetts Medical Society. 
PPROXIMATELY 15 TO 20\% OF BREAST cancers overexpress human epidermal growth factor receptor 2 (HER2). ${ }^{1,2}$ Despite dramatic therapeutic advances over the past 20 years, most patients with HER2-positive metastatic breast cancer ultimately die from their disease. ${ }^{3,4}$ Moreover, as systemic therapies have improved, the incidence of brain metastases, for which effective treatment options are limited, has increased such that brain metastases may develop in up to half of patients..$^{5-9}$ Standard-ofcare treatment for patients with HER2-positive metastatic breast cancer is first-line trastuzumab plus pertuzumab and a taxane, followed by second-line trastuzumab emtansine for patients who have disease progression. ${ }^{10-12}$ After progression during treatment with trastuzumab emtansine, no single regimen is considered the standard of care; commonly used regimens include tyrosine kinase inhibitors such as lapatinib with trastuzumab or capecitabine, trastuzumab with chemotherapy, or participation in a clinical trial.

Treatment for brain metastases in patients with HER2-positive breast cancer includes local therapies, such as neurosurgical resection and stereotactic (or whole-brain) radiation therapy. ${ }^{13}$ Although limited antitumor activity in the brain has been reported for some systemic HER2-targeted agents, including tyrosine kinase inhibitors and chemotherapy, ${ }^{14-18}$ data from randomized trials showing better treatment outcomes in these patients are lacking.

Tucatinib is an investigational, oral tyrosine kinase inhibitor that is highly selective for the kinase domain of HER2 with minimal inhibition of epidermal growth factor receptor, which may alter the toxicity profile. ${ }^{19,20}$ In a phase $1 \mathrm{~b}$ doseescalation trial, tucatinib in combination with trastuzumab and capecitabine showed encouraging antitumor activity in patients with HER2positive metastatic breast cancer, including those with brain metastases. This regimen was also associated with diarrhea, nausea, palmar-plantar erythrodysesthesia syndrome, fatigue, and vomiting; however, each grade 3 or higher treatmentrelated toxic effect (fatigue, diarrhea, and palmarplantar erythrodysesthesia syndrome) occurred in no more than $10 \%$ of patients. ${ }^{21}$ The HER2CLIMB trial evaluated tucatinib combined with trastuzumab and capecitabine in patients with HER2positive metastatic breast cancer previously treated with trastuzumab, pertuzumab, and trastuzumab emtansine.

\section{METHODS}

\section{TRIAL DESIGN AND OVERSIGHT}

We conducted an international, randomized, double-blind trial in which the combination of tucatinib plus trastuzumab and capecitabine was compared with placebo plus trastuzumab and capecitabine. The trial was conducted in accordance with regulatory requirements and International Conference on Harmonisation Good Clinical Practice guidelines. The protocol, available with the full text of this article at NEJM.org, was approved by institutional review boards and ethics committees, according to the practice at each participating trial site. All patients provided written informed consent. A steering committee provided scientific advice, and an independent data and safety monitoring committee oversaw the conduct of the trial. The steering committee and representatives of the sponsor (Seattle Genetics) designed the trial. The authors wrote the manuscript with the assistance of a medical writer funded by the sponsor. All the authors had full access to the relevant data, vouch for the completeness and accuracy of the data and for adherence of the trial to the protocol, and had final responsibility for the content of the manuscript and for the decision to submit the manuscript for publication.

\section{PATIENTS}

Patients 18 years of age or older were eligible to participate if they had advanced breast carcinoma that had been determined to be HER2-positive on the basis of immunohistochemical analysis, in situ hybridization, or fluorescence in situ hybridization and confirmed at a central location; had previously been treated with trastuzumab, pertuzumab, and trastuzumab emtansine; and had an Eastern Cooperative Oncology Group (ECOG) performance-status score of 0 or 1 (on a 5-point scale in which higher numbers reflect greater disability). Patients were excluded if they had previously received treatment for metastatic disease with capecitabine or a HER2-targeted tyrosine kinase inhibitor (although patients who had received lapatinib more than 12 months before initiating a trial regimen were eligible for 
inclusion). Patients with brain metastases were included unless they were in need of immediate local intervention, in which case they could receive local therapy and be enrolled subsequently. Patients with untreated brain metastases larger than $2 \mathrm{~cm}$ in diameter could be enrolled with approval from the medical monitor. Patients with leptomeningeal disease were excluded. A complete list of eligibility criteria is provided in the protocol.

\section{RANDOMIZATION AND TREATMENT}

Patients were randomly assigned in a 2:1 ratio to receive either tucatinib (300 $\mathrm{mg}$ orally twice daily throughout the treatment period) or placebo (orally twice daily), in combination with trastuzumab (6 mg per kilogram of body weight intravenously once every 21 days, with an initial loading dose of $8 \mathrm{mg}$ per kilogram; subcutaneous administration was allowed) and capecitabine (1000 mg per square meter of body-surface area orally twice daily on days 1 to 14 of each 21-day cycle) (Fig. S1 in the Supplementary Appendix, available at NEJM.org). Patients were stratified according to whether brain metastases were present (yes or no), ECOG performance-status score (0 or 1$)$, and geographic region (United States, Canada, or the rest of the world).

\section{ASSESSMENTS}

Contrast-enhanced spiral computed tomography (CT), positron-emission tomography-CT, or contrast-enhanced magnetic resonance imaging (MRI) (or combinations thereof) was performed at baseline, every 6 weeks for 24 weeks, and every 9 weeks thereafter. MRI of the head at baseline was required for all patients; those with brain metastases on the baseline scan underwent a contrast MRI of the head at the same intervals. Disease response and progression were evaluated in accordance with Response Evaluation Criteria in Solid Tumors (RECIST) criteria, version 1.1, ${ }^{22}$ by means of blinded independent central review. Laboratory assessments were performed at least every 3 weeks throughout the treatment period and 30 days after the last trial treatment date.

Safety was assessed on the basis of the incidence of adverse events (defined according to the Medical Dictionary for Regulatory Activities, version 22.0, and the National Cancer Institute Common Terminology Criteria for Adverse Events, version 4.03). Changes in vital signs and laboratory results were assessed in patients who received at least one dose of any trial drug or placebo.

\section{END POINTS}

The primary end point was progression-free survival (defined as the time from randomization to documented disease progression, as assessed by means of blinded independent central review, or death from any cause, whichever occurred first) in the first 480 patients who underwent randomization (primary end-point analysis population). Multiplicity-adjusted secondary end points were assessed in the total population and included overall survival (defined as the time from randomization to death from any cause); progression-free survival, as assessed by means of blinded independent central review, among the patients who had brain metastases at baseline; and confirmed objective response rate (defined as the percentage of patients with measurable disease at baseline who had a confirmed complete response or partial response, as assessed by means of blinded independent central review). Safety was a secondary end point.

\section{STATISTICAL ANALYSIS}

The primary end-point analysis was to be performed after approximately 288 events had occurred in the primary end-point analysis population, which would provide $90 \%$ power to detect a hazard ratio of 0.67 with a two-sided log-rank test at an alpha level of 0.05 . If the result with respect to the primary end point was significant, overall survival and progression-free survival among the patients with brain metastases were to be tested in parallel at alpha levels of 0.02 and 0.03 , respectively, in the first interim analysis. The final analyses for these two variables were to be performed with 361 and 220 events, respectively. Approximately 600 patients were to undergo randomization in order for the required number of events to be accumulated (see the Statistical Methods section in the Supplementary Appendix). Overall type I error in the interim and final analyses of overall survival in the total population and progression-free survival among the patients with brain metastases was controlled with the use of the group sequential Holm variable procedure ${ }^{23}$ with the Lan-DeMets alpha-spending function with an O'Brien-Fleming 
boundary. If the results with respect to both overall survival in the total population and progression-free survival among the patients with brain metastases were significant, the betweengroup difference in the percentage of patients who had a confirmed objective response was to be tested at a two-sided alpha level of 0.05 .

The size of the initial trial population (180 patients) was increased to 480 to provide adequate statistical power to evaluate the end point of progression-free survival and increase the power of the subgroup analyses. The size of the trial population was later increased to approximately 600 patients to ensure sufficient power to show a progression-free survival benefit among the patients with brain metastases. At no point were these decisions based on an assessment of the ongoing results, and blinding was never broken.

The Kaplan-Meier method was used to estimate progression-free survival and overall survival time curves, median progression-free survival and overall survival, and $95 \%$ confidence intervals for the treatment groups. Cox proportional-hazards models, with stratification factors taken into account, were used to estimate hazard ratios and $95 \%$ confidence intervals. The treatment groups were compared with the use of a stratified logrank test, and $\mathrm{P}$ values were calculated by means of a rerandomization procedure to reflect the dynamic, hierarchical randomization scheme. ${ }^{24}$ In the analysis of progression-free survival, data from patients without any documented event or who received anticancer therapy not specified in the protocol were censored at the date the patient was last known to be event-free. The censoring scheme for the primary end point is described in the statistical analysis plan (available with the protocol at NEJM.org). The P value for the between-group comparison of the percentage of patients who had a confirmed objective response was calculated with the use of a stratified Cochran-Mantel-Haenszel test.

At the date of data cutoff (September 4, 2019), a total of 275 events of disease progression or death had occurred in the primary end-point analysis population, 215 deaths had occurred in the total population, and 157 events of disease progression or death had occurred among the patients with brain metastases in the total population. On the basis of the observed number of events, the multiplicity-adjusted, two-sided alpha levels at the first interim analysis were 0.007 for overall survival and 0.008 for progression-free survival among the patients with brain metastases.

\section{RESULTS}

\section{PATIENT CHARACTERISTICS}

Between February 23, 2016, and May 3, 2019, a total of 612 patients were enrolled at 155 sites in 15 countries (total population); 410 were randomly assigned to the tucatinib-combination group and 202 to the placebo-combination group. In the primary end-point analysis population (480 patients), 320 patients were randomly assigned to the tucatinib-combination group and 160 to the placebo-combination group (Fig. S1 in the Supplementary Appendix). The median duration of follow-up in the total population was 14.0 months. Patient demographic and disease characteristics at baseline were well balanced between the treatment groups (Table 1). In the total population, 291 patients (47.5\%) had brain metastases at baseline - $48.3 \%$ in the tucatinibcombination group and $46.0 \%$ in the placebocombination group (Table S1).

\section{EFFICACY IN THE PRIMARY END-POINT POPULATION}

At 1 year, the estimated progression-free survival was $33.1 \%$ ( $95 \%$ confidence interval [CI], 26.6 to 39.7 ) in the tucatinib-combination group and $12.3 \%$ (95\% CI, 6.0 to 20.9 ) in the placebocombination group, and the median duration of progression-free survival was 7.8 months (95\% CI, 7.5 to 9.6) and 5.6 months (95\% CI, 4.2 to 7.1), respectively. The risk of disease progression or death, as assessed by means of blinded independent central review in the primary endpoint analysis population, was $46 \%$ lower in the tucatinib-combination group than in the placebo-combination group (hazard ratio, 0.54; $95 \%$ CI, 0.42 to 0.71 ; P<0.001) (Fig. 1A). Hazard ratios across all subgroups were consistent with the hazard ratio in the overall analysis (Fig. 1B). A comparison of investigator-assessed progression-free survival between the treatment groups yielded results consistent with those in the primary end-point analysis (Fig. S2).

\section{EFFICACY IN THE TOTAL TRIAL POPULATION}

At 2 years, the estimated overall survival was $44.9 \%$ (95\% CI, 36.6 to 52.8 ) in the tucatinibcombination group and 26.6\% (95\% CI, 15.7 to 38.7 ) in the placebo-combination group, and the median duration of overall survival was 21.9 


\begin{tabular}{|c|c|c|c|c|}
\hline \multirow[t]{2}{*}{ Characteristic } & \multicolumn{2}{|c|}{$\begin{array}{l}\text { Primary End-Point Analysis Population } \\
\qquad(\mathrm{N}=480)\end{array}$} & \multicolumn{2}{|c|}{$\begin{array}{l}\text { Total Population } \\
\quad(\mathrm{N}=612)\end{array}$} \\
\hline & $\begin{array}{l}\text { Tucatinib } \\
\text { Combination } \\
(\mathrm{N}=320)\end{array}$ & $\begin{array}{c}\text { Placebo } \\
\text { Combination } \\
(\mathrm{N}=160)\end{array}$ & $\begin{array}{l}\text { Tucatinib } \\
\text { Combination } \\
(\mathrm{N}=410)\end{array}$ & $\begin{array}{l}\text { Placebo } \\
\text { Combination } \\
(N=202)\end{array}$ \\
\hline Female sex — no. (\%) & $317(99.1)$ & $158(98.8)$ & 407 (99.3) & $200(99.0)$ \\
\hline \multicolumn{5}{|l|}{ Age - no. (\%) } \\
\hline$<65 \mathrm{yr}$ & $252(78.8)$ & $132(82.5)$ & $328(80.0)$ & $168(83.2)$ \\
\hline$\geq 65 \mathrm{yr}$ & $68(21.2)$ & $28(17.5)$ & $82(20.0)$ & $34(16.8)$ \\
\hline Median age - yr & 54.0 & 54.0 & 55.0 & 54.0 \\
\hline \multicolumn{5}{|l|}{ Race - no. (\%)† } \\
\hline Asian & $17(5.3)$ & $3(1.9)$ & $18(4.4)$ & $5(2.5)$ \\
\hline Black & $30(9.4)$ & $13(8.1)$ & $41(10.0)$ & $14(6.9)$ \\
\hline White & $225(70.3)$ & $125(78.1)$ & $287(70.0)$ & $157(77.7)$ \\
\hline Unknown or other & $48(15.0)$ & 19 (11.9) & $64(15.6)$ & $26(12.9)$ \\
\hline \multicolumn{5}{|l|}{ Geographic region — no. (\%) } \\
\hline United States and Canada & $204(63.8)$ & $103(64.4)$ & $246(60.0)$ & $123(60.9)$ \\
\hline Rest of the world & $116(36.2)$ & $57(35.6)$ & $164(40.0)$ & $79(39.1)$ \\
\hline \multicolumn{5}{|l|}{ Hormone-receptor status — no. (\%) } \\
\hline Positive for ER or PR or both & $190(59.4)$ & 99 (61.9) & $243(59.3)$ & $127(62.9)$ \\
\hline Negative for ER and PR & $126(39.4)$ & $61(38.1)$ & $161(39.3)$ & $75(37.1)$ \\
\hline Other & $4(1.2)$ & 0 & $6(1.5)$ & 0 \\
\hline \multicolumn{5}{|l|}{ ECOG performance-status score - no. $(\%) \ddagger$} \\
\hline 0 & $159(49.7)$ & $76(47.5)$ & $204(49.8)$ & $94(46.5)$ \\
\hline 1 & $161(50.3)$ & $84(52.5)$ & $206(50.2)$ & $108(53.5)$ \\
\hline Stage IV at initial diagnosis — no. (\%) & $108(33.8)$ & 67 (41.9) & $143(34.9)$ & $77(38.1)$ \\
\hline Presence or history of brain metastases — no. (\%) & $148(46.2)$ & $71(44.4)$ & $198(48.3)$ & $93(46.0)$ \\
\hline \multicolumn{5}{|l|}{ Location of other metastases - no. (\%) } \\
\hline Lung & $160(50.0)$ & $82(51.2)$ & $200(48.8)$ & $100(49.5)$ \\
\hline Liver & $108(33.8)$ & $64(40.0)$ & $137(33.4)$ & $78(38.6)$ \\
\hline Bone & $178(55.6)$ & $85(53.1)$ & $223(54.4)$ & $111(55.0)$ \\
\hline Previous lines of therapy, median no. (range) & $4(2-14)$ & $4(2-17)$ & $4(2-14)$ & $4(2-17)$ \\
\hline $\begin{array}{l}\text { Previous lines of therapy for metastatic cancer, median } \\
\text { no. (range) }\end{array}$ & $3(1-14)$ & $3(1-13)$ & $3(1-14)$ & $3(1-13)$ \\
\hline \multicolumn{5}{|l|}{ Previous therapies — no. (\%) } \\
\hline Trastuzumab & $320(100)$ & $160(100)$ & $410(100)$ & $202(100)$ \\
\hline Pertuzumab & $320(100)$ & $159(99.4)$ & $409(99.8)$ & $201(99.5)$ \\
\hline Trastuzumab emtansine & $320(100)$ & $160(100)$ & $410(100)$ & $202(100)$ \\
\hline Lapatinib & $22(6.9)$ & $10(6.2)$ & $24(5.9)$ & $10(5.0)$ \\
\hline
\end{tabular}

* The primary end-point analysis population included the first 480 patients who were randomly assigned to the tucatinib-combination group (tucatinib plus trastuzumab and capecitabine) or to the placebo-combination group (placebo plus trastuzumab and capecitabine), and the total population included 612 patients who underwent randomization. Randomization stratification factors included geographic region (United States, Canada, or the rest of the world), presence or history of brain metastases (yes or no), and Eastern Cooperative Oncology Group (ECOG) performance-status score $(0$ or 1$)$. ER denotes estrogen receptor, and PR progesterone receptor. Data from the patients in the United States and Canada were combined for this analysis.

$\uparrow$ Race was determined by the local investigator.

$\checkmark$ ECOG performance-status scores range from 0 to 5 , with higher scores indicating greater disability. 


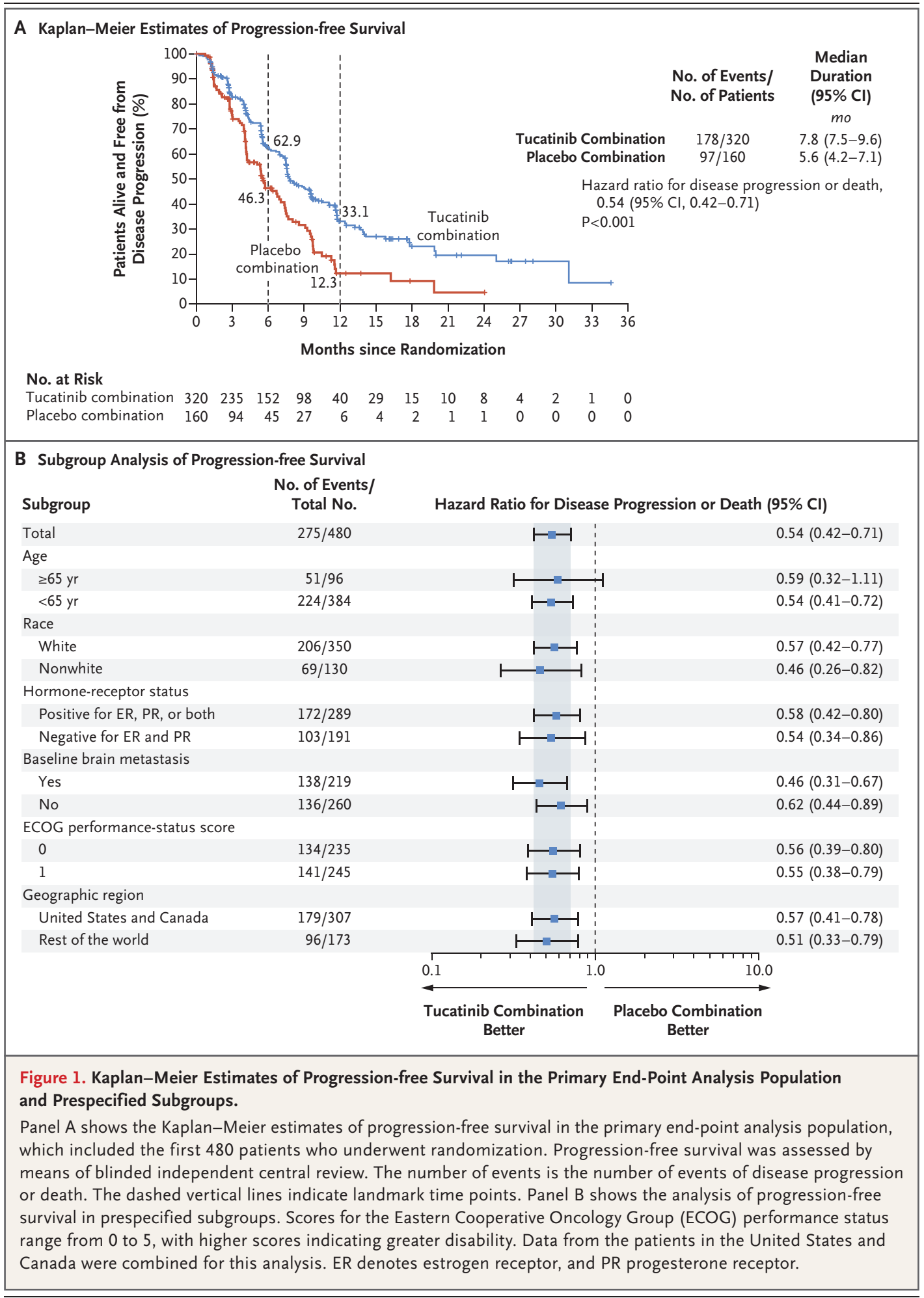




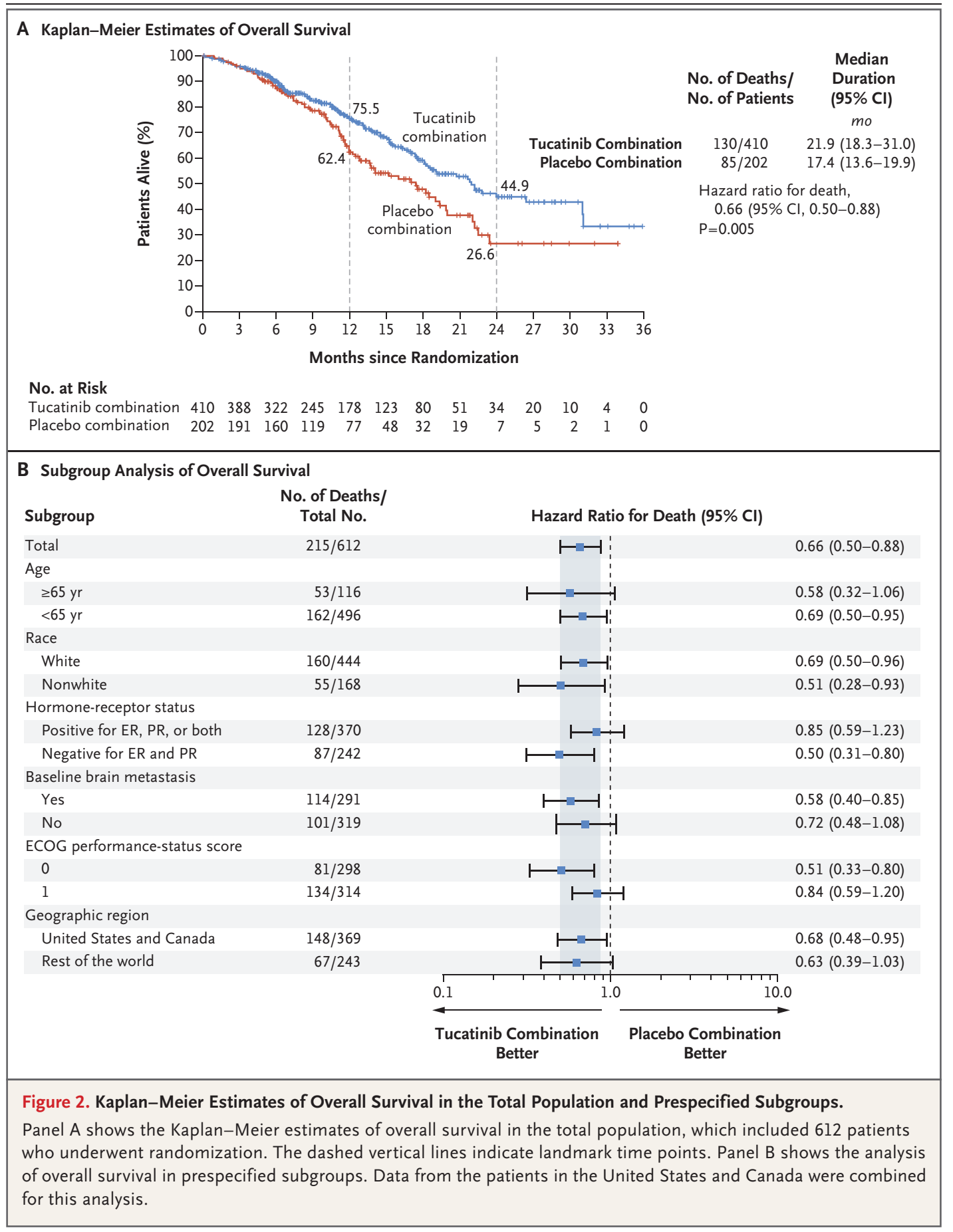

months (95\% CI, 18.3 to 31.0) and 17.4 months (95\% CI, 13.6 to 19.9), respectively. In the total population, the risk of death was 34\% lower in the tucatinib-combination group than in the placebo-combination group (hazard ratio, 0.66; 95\% CI, 0.50 to $0.88 ; \mathrm{P}=0.005$ ) (Fig. 2A). Hazard ratios across all subgroups were consistent with the hazard ratio in the overall analysis (Fig. 2B). In addition, progression-free survival in the total population was consistent with that in the primary end-point analysis (Fig. S3).

Among the patients with brain metastases, 
the estimated progression-free survival at 1 year was $24.9 \%$ (95\% CI, 16.5 to 34.3 ) in the tucatinib-combination group and $0 \%$ in the placebocombination group, and the median duration of progression-free survival was 7.6 months $(95 \%$ CI, 6.2 to 9.5) and 5.4 months (95\% CI, 4.1 to 5.7), respectively. The risk of disease progression or death was $52 \%$ lower in the tucatinib-combination group than in the placebo-combination group (hazard ratio, 0.48 ; $95 \% \mathrm{CI}, 0.34$ to 0.69 ; $\mathrm{P}<0.001$ ) (Fig. 3A). Hazard ratios across all subgroups were consistent with the hazard ratio in the overall analysis (Fig. 3B). In a prespecified analysis involving the patients without brain metastases, the risk of disease progression or death was $43 \%$ lower in the tucatinib-combination group than in the placebo-combination group (hazard ratio, 0.57; 95\% CI, 0.41 to 0.80 ) (Fig. S4). Among the 511 patients with measurable disease at baseline, as assessed by means of blinded independent central review, the percentage who had a confirmed objective response was $40.6 \%$ ( $95 \%$ CI, 35.3 to 46.0 ) in the tucatinib-combination group and $22.8 \%$ (95\% CI, 16.7 to 29.8) in the placebo-combination group $(\mathrm{P}<0.001)$ (Table $\mathrm{S} 2)$.

\section{TREATMENT DURATION}

In the primary end-point analysis population, the median duration of exposure to tucatinib was 7.3 months (range, $<0.1$ to 35.1 ), and the median duration of exposure to placebo was 4.4 months (range, $<0.1$ to 24.0); the durations of exposure to trastuzumab and capecitabine were also shorter in the placebo-combination group. Among the 601 patients who received at least one dose of any trial drug or placebo, the median duration of exposure to tucatinib or placebo was 5.8 months (range, $<0.1$ to 35.1 ) and 4.4 months (range, $<0.1$ to 24.0 ), respectively. At the date of data cutoff, 118 of 410 patients (28.8\%) in the tucatinib-combination group and 27 of 202 $(13.4 \%)$ in the placebo-combination group were continuing to receive their assigned treatment.

\section{SAFETY}

The occurrence of adverse events according to treatment group is summarized in Table 2 and Table S3. Adverse events led to the discontinuation of tucatinib in $5.7 \%$ of the patients, to the discontinuation of placebo in $3.0 \%$ of the patients (Table S4), and to the discontinuation of capecitabine in $9.8 \%$ of the patients $(10.1 \%$ in the tucatinib-combination group and $9.1 \%$ in the placebo-combination group).

The most common adverse events observed among the patients in the tucatinib-combination group were diarrhea, palmar-plantar erythrodysesthesia syndrome, nausea, fatigue, and vomiting (Table 2); most events were of grade 1 or 2. The most common adverse events of grade 3 or higher observed among the patients in the tucatinib-combination group were palmar-plantar erythrodysesthesia syndrome, diarrhea, elevations in alanine aminotransferase (ALT) and aspartate aminotransferase (AST) levels, and fatigue.

Diarrhea was the most common adverse event in both the tucatinib-combination group and the placebo-combination group, and most events of diarrhea were of grade 1 (in $43.3 \%$ and $32.0 \%$ of the patients, respectively) or grade 2 (in $24.8 \%$ and $12.7 \%$, respectively); diarrhea of grade 3 or higher occurred in $12.9 \%$ and $8.6 \%$, respectively. Antidiarrheal prophylaxis was not mandated. Among the patients who used antidiarrheal agents, the median duration of use was 3 days per cycle in both treatment groups.

Elevations in the ALT and AST levels were mostly low-grade, transient, and reversible; ALT and AST elevations that were of grade 3 or higher occurred in $5.4 \%$ and $4.5 \%$ of the patients, respectively, in the tucatinib-combination group and in $0.5 \%$ of the patients (for each) in the placebo-combination group. Elevations in the bilirubin level of any grade occurred in $18.6 \%$ of the patients in the tucatinib-combination group and in $10.2 \%$ of those in the placebo-combination group; however, elevations of grade 3 or higher occurred in fewer patients in the tucatinib-combination group ( $0.7 \%$ vs. $2.5 \%)$.

Increases in the serum creatinine level were observed in $13.9 \%$ of the patients in the tucatinib-combination group and in $1.5 \%$ of those in the placebo-combination group. Tucatinib has been shown to inhibit the multidrug and toxin extrusion protein 1 and 2-K (MATE1 and MATE2-K) transporters, which increases the serum creatinine level without affecting glomerular function (see the Supplementary Appendix). Increases in the serum creatinine level occurred early, remained clinically nonsignificant during the treatment period with no intervention to lower the level, were reversible, and were not the cause of discontinuation of therapy in any patient.

Of 215 deaths that occurred during the trial, 


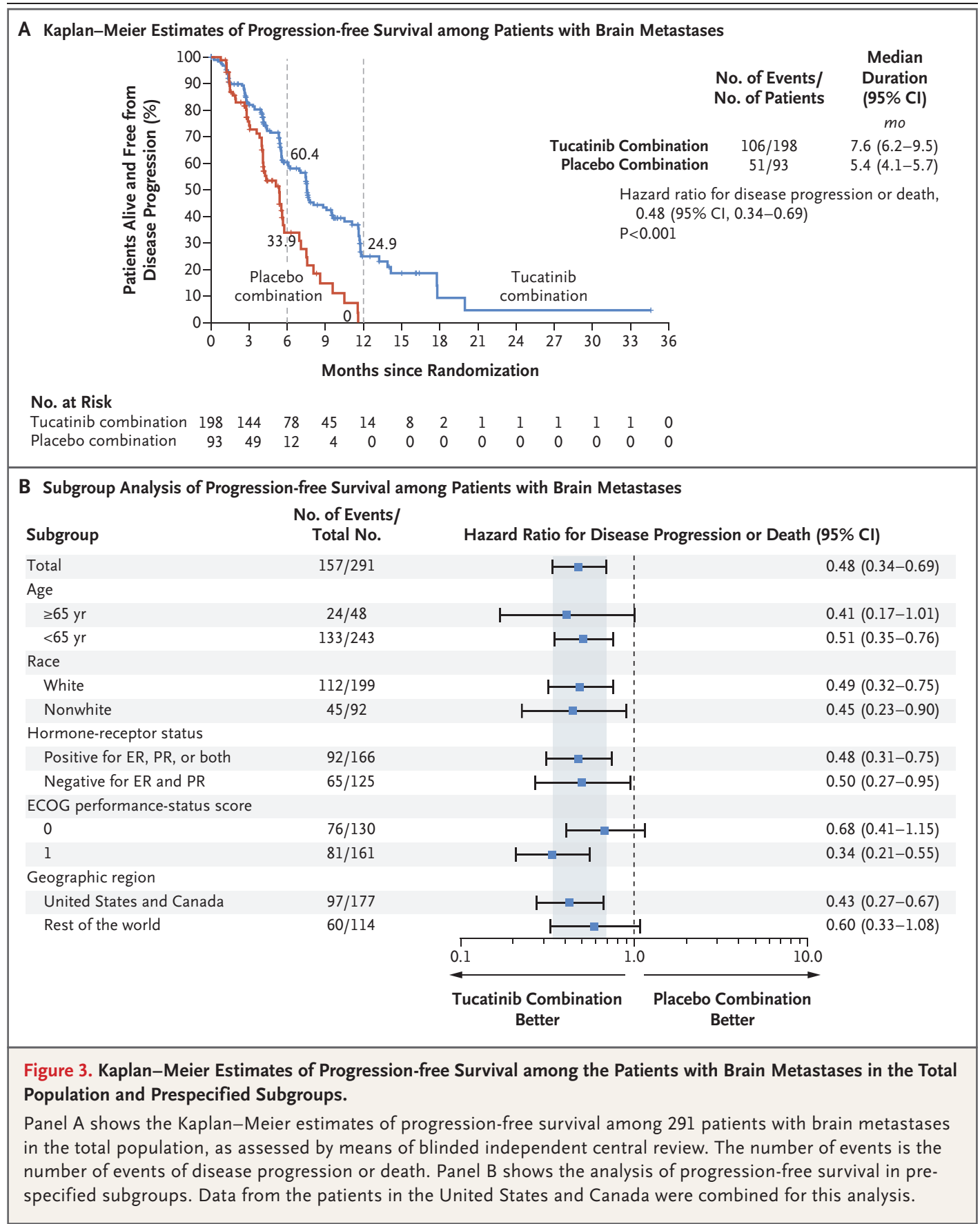

the most common cause in both treatment groups was disease progression. Adverse events were the cause of death in 6 of 404 patients $(1.5 \%)$ in the tucatinib-combination group (cardiac arrest, cardiac failure, dehydration, multipleorgan dysfunction syndrome, sepsis, and septic shock in 1 patient each) and in 5 of 197 patients $(2.5 \%)$ in the placebo-combination group (cardiac arrest, multiple-organ dysfunction syndrome, myocardial infarction, sepsis, and systemic inflammatory response syndrome in 1 patient each).

\section{DISCUSSION}

Among patients with HER2-positive metastatic breast cancer previously treated with trastuzu- 


\begin{tabular}{|c|c|c|c|c|}
\hline \multirow[t]{3}{*}{ Event } & \multicolumn{2}{|c|}{$\begin{array}{l}\text { Tucatinib-Combination Group } \\
\qquad(\mathrm{N}=404)\end{array}$} & \multicolumn{2}{|c|}{$\begin{array}{l}\text { Placebo-Combination Group } \\
\qquad(\mathrm{N}=197)\end{array}$} \\
\hline & Any Grade & Grade $\geq 3$ & Any Grade & Grade $\geq 3$ \\
\hline & \multicolumn{4}{|c|}{ number of patients (percent) } \\
\hline Any adverse event & $401(99.3)$ & $223(55.2)$ & $191(97.0)$ & $96(48.7)$ \\
\hline Diarrhea & $327(80.9)$ & $52(12.9)$ & $105(53.3)$ & $17(8.6)$ \\
\hline PPE syndrome & $256(63.4)$ & $53(13.1)$ & $104(52.8)$ & $18(9.1)$ \\
\hline Nausea & $236(58.4)$ & $15(3.7)$ & $86(43.7)$ & $6(3.0)$ \\
\hline Fatigue & $182(45.0)$ & $19(4.7)$ & $85(43.1)$ & $8(4.1)$ \\
\hline Vomiting & $145(35.9)$ & $12(3.0)$ & $50(25.4)$ & $7(3.6)$ \\
\hline Stomatitis & $103(25.5)$ & $10(2.5)$ & $28(14.2)$ & $1(0.5)$ \\
\hline Decreased appetite & $100(24.8)$ & $2(0.5)$ & $39(19.8)$ & 0 \\
\hline Headache & $87(21.5)$ & $2(0.5)$ & $40(20.3)$ & $3(1.5)$ \\
\hline $\begin{array}{l}\text { Aspartate aminotransferase in- } \\
\text { creased }\end{array}$ & $86(21.3)$ & $18(4.5)$ & $22(11.2)$ & $1(0.5)$ \\
\hline $\begin{array}{l}\text { Alanine aminotransferase in- } \\
\text { creased }\end{array}$ & $81(20.0)$ & $22(5.4)$ & $13(6.6)$ & $1(0.5)$ \\
\hline
\end{tabular}

mab, pertuzumab, and trastuzumab emtansine, the addition of tucatinib to trastuzumab and capecitabine resulted in a clinically meaningful lower risk of disease progression or death than the addition of placebo. Most importantly, overall survival was longer by 4.5 months with tucatinib (median of 21.9 months vs. 17.4 months).

The tucatinib combination was associated with a significantly lower risk of disease progression or death than the placebo combination among patients with metastatic HER2-positive breast cancer with brain metastases. Previous studies have shown that the combination of a HER2 tyrosine kinase inhibitor with capecitabine has some activity in brain metastases from HER2-positive breast cancer. ${ }^{17,25}$ However, toxic effects, including those associated with inhibition of epidermal growth factor receptor, have limited the use of these combinations.

The survival benefit with tucatinib was observed in the total HER2CLIMB trial population and across all subgroups tested. In addition, the results showed that, in combination with capecitabine, simultaneous targeting of the internal domain of HER2, as well as the external domain with trastuzumab, led to substantially better survival outcomes than did targeting the external domain alone.

Patients were enrolled across 155 sites in 15 countries, and all patients had received previous treatment with the current standard of care (trastuzumab, pertuzumab, and trastuzumab emtansine). The comparator group received a recommended regimen for management in this clinical context, as supported by a randomized, phase 3 trial that showed longer progressionfree and overall survival among patients treated with trastuzumab plus capecitabine than among those treated with lapatinib plus capecitabine. ${ }^{26}$ Survival outcomes in the placebo-combination group in our trial were similar to those in other contemporary trials of HER2-targeted therapy. ${ }^{25,27}$

In our trial, we enrolled a large percentage of patients with untreated or previously treated progressing brain metastases, a population typically excluded from clinical trials despite this condition being a common clinical problem. The inclusion of patients with brain metastases in the initial phase 1 trials of tucatinib generated preliminary efficacy data to support their inclu- 
sion in HER2CLIMB and is in line with current recommendations from the American Society of Clinical Oncology-Friends of Cancer Research Eligibility Criteria Working Group. ${ }^{28}$

Tucatinib in combination with trastuzumab and capecitabine was associated with toxic effects, with the majority of adverse events being low grade. Safety events of note included diarrhea that was managed with short courses of antidiarrheal agents and transient, reversible elevations in liver enzyme levels. A majority of patients in the tucatinib-combination group had toxic effects, but less than $6 \%$ of the patients discontinued treatment with tucatinib as a consequence.

In conclusion, tucatinib plus trastuzumab and capecitabine is an active combination in heavily pretreated patients with HER2-positive metastatic breast cancer, including those with previously untreated, treated and stable, or treated and progressing brain metastases.

A data sharing statement provided by the authors is available with the full text of this article at NEJM.org.

Supported by Seattle Genetics.

Disclosure forms provided by the authors are available with the full text of this article at NEJM.org.

We thank the patients who participated in this trial and their families, as well as the investigators and staff at all HER2CLIMB clinical sites; the members of the independent data and safety monitoring committee and the independent review committee; Andres Forero-Torres, M.D., and Matthew Blahna, Ph.D., Seattle Genetics, for critical review and revision of the manuscript; Laurie LaRusso, M.S., Chestnut Medical Communications, for writing support during the development of the manuscript; Jorge Ramos, D.O., JoAl Mayor, Pharm.D., B.C.O.P., and Suzanne McGoldrick, M.D., for the medical monitoring of the trial; and the entire HER2CLIMB trial team for their diligent data review.

\section{APPENDIX}

The authors' full names and academic degrees are as follows: Rashmi K. Murthy, M.D., Sherene Loi, M.D., Alicia Okines, M.D., Elisavet Paplomata, M.D., Erika Hamilton, M.D., Sara A. Hurvitz, M.D., Nancy U. Lin, M.D., Virginia Borges, M.D., Vandana Abramson, M.D., Carey Anders, M.D., Philippe L. Bedard, M.D., Mafalda Oliveira, M.D., Erik Jakobsen, M.D., Thomas Bachelot, M.D., Shlomit S. Shachar, M.D., Volkmar Müller, M.D., Sofia Braga, M.D., Francois P. Duhoux, M.D., Richard Greil, M.D., David Cameron, M.D., Lisa A. Carey, M.D., Giuseppe Curigliano, M.D., Ph.D., Karen Gelmon, M.D., Gabriel Hortobagyi, M.D., Ian Krop, M.D., Ph.D., Sibylle Loibl, M.D., Mark Pegram, M.D., Dennis Slamon, M.D., M. Corinna Palanca-Wessels, M.D., Ph.D., Luke Walker, M.D., Wentao Feng, Ph.D., and Eric P. Winer, M.D.

The authors' affiliations are as follows: M.D. Anderson Cancer Center, Houston (R.K.M., G.H.); Peter MacCallum Cancer Centre, Melbourne, VIC, Australia (S. Loi); the Royal Marsden NHS Foundation Trust, London (A.O.), and Edinburgh Cancer Research Centre, Edinburgh (D.C.) — both in the United Kingdom; Winship Cancer Institute, Atlanta (E.P.); Sarah Cannon Research Institute/Tennessee Oncology-Nashville (E.H.) and Vanderbilt University Medical Center (V.A.), Nashville; University of California, Los Angeles, Medical Center-Jonsson Comprehensive Cancer Center, Los Angeles (S.A.H., D.S.), and Stanford Comprehensive Cancer Institute, Palo Alto (M.P.) - both in California; Dana-Farber Cancer Institute, Boston (N.U.L., I.K., E.P.W.); University of Colorado Cancer Center, Aurora (V.B.); Duke Cancer Institute, Durham (C.A.), and University of North Carolina Lineberger Comprehensive Cancer Center, Chapel Hill (L.A.C.) — both in North Carolina; University Health Network, Princess Margaret Cancer Centre, Toronto (P.L.B.), and British Columbia Cancer, Vancouver (K.G.) — both in Canada; Hospital Universitario Vall D'Hebron, Barcelona (M.O.); Sygehus LillebaeltVejle Sygehus, Vejle, Denmark (E.J.); Centre Léon Bérard, Lyon, France (T.B.); Rambam Health Care Campus, Haifa, Israel (S.S.S.); Universitaetsklinikum Hamburg-Eppendorf, Hamburg (V.M.), and German Breast Group, Neu-Isenburg (S. Loibl) — both in Germany; Hospital Cuf Descobertas R. Mário Botas, Lisbon, Portugal (S.B.); Cliniques Universitaires Saint-Luc, Brussels (F.P.D.); Third Medical Department, Paracelsus Medical University Salzburg, Salzburg Cancer Research Institute-Center for Clinical Cancer and Immunology Trials, and Cancer Cluster Salzburg, Salzburg, Austria (R.G.); Istituto Europeo di Oncologia, IRCCS, University of Milan, Milan (G.C.); and Seattle Genetics, Bothell, WA (M.C.P.-W., L.W., W.F.).

\section{REFERENCES}

1. Owens MA, Horten BC, Da Silva MM. HER2 amplification ratios by fluorescence in situ hybridization and correlation with immunohistochemistry in a cohort of 6556 breast cancer tissues. Clin Breast Cancer 2004;5:63-9.

2. Cronin KA, Harlan LC, Dodd KW, Abrams JS, Ballard-Barbash R. Population-based estimate of the prevalence of HER-2 positive breast cancer tumors for early stage patients in the US. Cancer Invest 2010;28:963-8.

3. Baselga J, Cortés J, Kim S-B, et al. Pertuzumab plus trastuzumab plus docetaxel for metastatic breast cancer. N Engl J Med 2012;366:109-19.

4. Swain SM, Kim SB, Cortés J, et al. Pertuzumab, trastuzumab, and docetaxel for
HER2-positive metastatic breast cancer (CLEOPATRA study): overall survival results from a randomised, double-blind, placebo-controlled, phase 3 study. Lancet Oncol 2013;14:461-71.

5. Bendell JC, Domchek SM, Burstein $\mathrm{HJ}$, et al. Central nervous system metastases in women who receive trastuzumabbased therapy for metastatic breast carcinoma. Cancer 2003;97:2972-7.

6. Brufsky AM, Mayer M, Rugo HS, et al. Central nervous system metastases in patients with HER2-positive metastatic breast cancer: incidence, treatment, and survival in patients from registHER. Clin Cancer Res 2011;17:4834-43.

7. Leyland-Jones B. Human epidermal growth factor receptor 2-positive breast cancer and central nervous system metastases. J Clin Oncol 2009;27:5278-86.

8. Olson EM, Najita JS, Sohl J, et al. Clinical outcomes and treatment practice patterns of patients with HER2-positive metastatic breast cancer in the posttrastuzumab era. Breast 2013;22:525-31.

9. Pestalozzi BC, Holmes E, de Azambuja E, et al. CNS relapses in patients with HER2-positive early breast cancer who have and have not received adjuvant trastuzumab: a retrospective substudy of the HERA trial (BIG 1-01). Lancet Oncol 2013;14:244-8.

10. National Comprehensive Cancer Network (NCCN). Clinical practice guidelines in oncology: breast cancer. Version 3.2018. October 25, 2018 (http://www 
.nccn.org/professionals/physician_gls/pdf/ breast.pdf).

11. Giordano SH, Temin S, Davidson NE. Systemic therapy for patients with advanced human epidermal growth factor receptor 2-positive breast cancer: ASCO clinical practice guideline update summary. J Oncol Pract 2018;14:501-4.

12. Cardoso F, Senkus E, Costa A, et al. 4th ESO-ESMO international consensus guidelines for advanced breast cancer (ABC 4). Ann Oncol 2018;29:1634-57.

13. National Comprehensive Cancer Network (NCCN). Clinical practice guidelines in oncology: central nervous system cancers - breast cancer. Version 3.2019. October 18, 2019 (https://www.nccn.org/ professionals/physician_gls/pdf/cns.pdf). 14. Lin NU, Diéras V, Paul D, et al. Multicenter phase II study of lapatinib in patients with brain metastases from HER2positive breast cancer. Clin Cancer Res 2009;15:1452-9.

15. Sutherland S, Ashley S, Miles D, et al. Treatment of HER2-positive metastatic breast cancer with lapatinib and capecitabine in the lapatinib expanded access programme, including efficacy in brain metastases - the UK experience. $\mathrm{Br} \mathrm{J}$ Cancer 2010;102:995-1002.

16. Bachelot T, Romieu G, Campone M, et al. Lapatinib plus capecitabine in patients with previously untreated brain metastases from HER2-positive metastatic breast cancer (LANDSCAPE): a singlegroup phase 2 study. Lancet Oncol 2013; 14:64-71.
17. Freedman RA, Gelman RS, Anders CK, et al. TBCRC 022: a phase II trial of neratinib and capecitabine for patients with human epidermal growth factor receptor 2-positive breast cancer and brain metastases. J Clin Oncol 2019;37:1081-9. 18. Krop IE, Lin NU, Blackwell $\mathrm{K}$, et al. Trastuzumab emtansine (T-DM1) versus lapatinib plus capecitabine in patients with HER2-positive metastatic breast cancer and central nervous system metastases: a retrospective, exploratory analysis in EMILIA. Ann Oncol 2015;26:113-9.

19. Moulder SL, Borges VF, Baetz T, et al Phase I study of ONT-380, a HER2 inhibitor, in patients with HER $2^{+}$-advanced solid tumors, with an expansion cohort in HER2 metastatic breast cancer (MBC). Clin Cancer Res 2017;23:3529-36.

20. Pheneger T, Bouhana $K$, Anderson $D$, et al. In vitro and in vivo activity of ARRY380: a potent, small molecule inhibitor of ErbB2. Cancer Res 2009;69:1795. abstract. 21. Murthy R, Borges VF, Conlin A, et al Tucatinib with capecitabine and trastuzumab in advanced HER2-positive metastatic breast cancer with and without brain metastases: a non-randomised, open-label, phase 1b study. Lancet Oncol 2018;19: 880-8.

22. Eisenhauer EA, Therasse P, Bogaerts J, et al. New response evaluation criteria in solid tumours: revised RECIST guideline (version 1.1). Eur J Cancer 2009;45:22847.

23. Ye Y, Li A, Liu L, Yao B. A group sequential Holm procedure with multiple primary endpoints. Stat Med 2013;32:111224.

24. Rosenberger WF, Lachin JM. Randomization in clinical trials: theory and practice. Hoboken, NJ: John Wiley and Sons, 2016.

25. Saura C, Oliveira M, Feng Y-H, et al. Neratinib + capecitabine versus lapatinib + capecitabine in patients with HER2+ metastatic breast cancer previously treated with $\geq 2$ HER2-directed regimens: findings from the multinational, randomized, phase III NALA trial. J Clin Oncol 2019;37:1002. abstract.

26. Pivot X, Manikhas A, Żurawski B, et al. CEREBEL (EGF111438): a phase III, randomized, open-label study of lapatinib plus capecitabine versus trastuzumab plus capecitabine in patients with human epidermal growth factor receptor 2-positive metastatic breast cancer. J Clin Oncol 2015;33:1564-73.

27. Rugo HS, Im S-A, Wright GLS, et al. SOPHIA primary analysis: a phase 3 (P3) study of margetuximab $(M)+$ chemotherapy (C) versus trastuzumab (T) $+\mathrm{C}$ in patients (pts) with HER2+ metastatic (met) breast cancer (MBC) after prior anti-HER2 therapies (Tx). J Clin Oncol 2019;37:1000. abstract.

28. Lin NU, Prowell T, Tan AR, et al. Modernizing clinical trial eligibility criteria: recommendations of the American Society of Clinical Oncology-Friends of Cancer Research Brain Metastases Working Group. J Clin Oncol 2017;35:3760-73. Copyright () 2019 Massachusetts Medical Society. 